\title{
BOARD OF DIRECTORS
}

\section{OFFICERS}

Honorary President

President

Past President

Second Vice-President

Treasurer

Corresponding Secretary

Recording Secretary

\section{APPOINTED DIRECTORS}

Blue Jay Editor

Blue Jay Workshop

Circulation

Conservation

Endangered Species

Grasslands Park

Local Societies

Elizabeth Cruickshank

Gary W. Seib

Margaret Belcher

Ole Nielsen

Frank Switzer

George R. Dodd

Gary Wobeser

Dale Hjertaas

J. Bernard Gollop

Frank Brazier

Lorne Scott

Frank Switzer

1301 Shannon Rd., Regina, S4S 5K9

James R. Jowsey 2635 Nineteenth Ave., Regina, S4T 1 X2 George F. Ledingham 2335 Athol St., Regina, S4T 3G4 Lorne Scott

Saskatchewan Museum of Natural History, Regina Lynn Oliphant

Lloyd O. T. Peterson

Wm. and Joyce Anaka

C. Stuart Houston 863 University Dr., Saskatoon, S7N 0J8
Membership

Newsletter Editors

Special Publications
2339 Athol St., Regina, S4T 3G4 2-2104 14th Ave., Regina, S4P 0X6 2601 Winnipeg St., Regina, S4P 1 H8 1301 Shannon Rd., Regina, S4S 5K9 33 Malone Cresc., Regina, S4S 5R 1 209 Tucker Cresc., Saskatoon, S7H 3J1 1317 Osler St., Saskatoon, S7N 0V1

2202 York Ave., Saskatoon, S7J 1J1 2657 Cameron St., Regina, S4T 2W5
First Vice-President

Saskatchewan Museum of Natural History, Regina

\section{REPRESENTATIVES AT LARGE}

Ron E. Andrews

843 Athabasca St. W., Moose Jaw, Sask. S6H 2 E2 73 Cardinal Cresc., Regina, Sask. S4S 4 Y6 Broadview, Sask. SOG OKO Nokomis, Sask. SOG 3RO

Box 93, Raymore, Sask. SOA 3J0

Box 839, Wolseley, Saks. SOG $5 \mathrm{HO}$ Dept. of Zoology, Univ. of Alberta, Edmonton, Alta. T6G 2E1 Box 117, Waseca, Sask. SOM 3A0 Box 2103, Nipawin, Sask. SOE 1 E0 Dept. of Zoology, Univ. of Manitoba, Winnipeg, Man. R3T 2 O2 209 Tucker Cresc., Saskatoon, Sask. S7H 3 J 1

\section{PRESIDENTS OF LOCAL SOCIETIES}

Fort Qu'Appelle

Indian Head

Maple Creek

Moose Jaw

Saskatoon

Swift Currer

U
Yorkton

University Biology Clubs
Bernard de Vries

Mary Skinner

D. Bromley

Patricia Kern

W. Lloyd Hipperson

Lynn Oliphant

Jan Looman

Richard Collins

Phil Pawluck

Richard Collins

Dale Hjertaas
Box 1043, S0G ISO P.O. Box 777, SOG $2 \mathrm{KO}$ SON INO

1053 Chestnut Ave., S6H 1 A 7 3708 Albert St., S4S 3 P9 330 Saskatchewan Cresc. W., S7M 0A4

491 - 2nd Ave. S.E., S9H 3 J 7

Regina Campus, S4S 0A2

163 Peaker Ave., S3N 1 S6

University of Regina, S4S $0 \mathrm{~A} 2$

University of Saskatchewan, S7N OWO 